\title{
Theory of Adaptive Adjustment
}

\author{
WEIHONG HUANG* \\ Nanyang Business School, Nanyang Technological University, Nanyang Avenue, Singapore 639798
}

(Received 7 May 2000)

\begin{abstract}
Conventional adaptive expectation as a mechanism of stabilizing an unstable economic process is reexamined through a generalization to an adaptive adjustment framework. The generic structures of equilibria that can be stabilized through an adaptive adjustment mechanism are identified. The generalization can be applied to a broad class of discrete economic processes where the variables interested can be adjusted or controlled directly by economic agents such as in cobweb dynamics, Cournot games, Oligopoly markets, tatonnement price adjustment, tariff games, population control through immigration etc.
\end{abstract}

Keywords: Adaptive expectations; Adaptive adjustment; Chaos; Nonlinear dynamics

\section{MOTIVATIONS}

Adaptive expectations, as one of the main backward-looking expectations, has long been utilized by economists to stabilize a dynamical economic process. Early in Nerlove (1958), it was shown that a traditional linear cobweb model with naive expectations could be stabilized, should adaptive expectations be introduced. Since then, numerous studies on the effects of adaptive expectations on the stability of economic equilibria have emerged under different contexts such as oligopoly markets (Okuguchi, 1970, Okuguchi and Szidarovszky, 1990) and adaptive learning (Marcet and Sargent, 1986) etc. Most studies are concerned either with the comparison between the consequences of adaptive expectations and rational expectations, or with the stability conditions of equilibria under some specific model specifications (Fisher, 1961).

Along with the prevalence of the forwardlooking expectations such as the rational expectations hypothesis since the 1960's, the adaptive expectations has become gradually outdated (Mills, 1961). It wasn't until the emergence of nonlinear dynamics of chaos in the late seventies that adaptive expectations once again attracted the attention of economists. For instances, Heiner $(1989,1992)$ showed that the adaptive adjustment of decision variables can lead to their convergence to optimal targets under a general decision-making framework. Chiarella (1988) and Hommes (1991) observed that a Cobweb model with adaptive

*e-mail: awhhuang@,ntu.edu.sg 
expectations and nonlinear but monotonic supply and demand curves may lead to chaotic fluctuations. Other related studies can be found in Naish (1993); Conlisk (1993); Garratt (1997) and Onozaki et al. (2000). Recently applications to duopoly models are provided by Puu (1997, 1998), oligopoly market of multiproducts are given by Szidarovszky and Weiye (2000) and Rassenti et al. (2000).

Theoretically, even though the adaptive expectation can be ensured to equate its realized counterpart in equilibrium, the convergence to such an equilibrium can not be guaranteed, especially in the case of a nonlinear multi-variables process. It is the aim of this article to reconsider an adaptive expectations scheme as a means of stabilizing a nonlinear economic process from an angle different from conventional studies. Instead of focusing the stability conditions under some specific economic processes, we shall turn to exploring the internal dynamical structure of an economic process that can be stabilized through adaptive expectations under a general framework.

To furnish this purpose and to have a broader view about the rationale and functioning of adaptive expectations, we proceed by generalizing the concept of adaptive expectations to adaptive adjustment mechanism in such a way that economic variables are directly adjusted adaptively to their equilibrium. More specifically, for an endogenous economic variable, say price, $p_{t}$, adaptive expectation in economics means that the expected price for next period $p_{t+1}^{e}$ is formed iteratively (dynamically) through weighted averaging of current period's expectation (formed in last period) with current period's realized price as follows: $p_{t+1}^{e}=(1-\alpha) p_{t}^{e}+\alpha p t$, where $\alpha$ is commonly referred to as an adjustment parameter, and is assumed to be in the range of zero and unity. By adaptive adjustment, however, we mean to purposely modify a discrete economic process $x_{t+1}=$ $\theta\left(x_{t}\right)$ into $x_{t+1}=(1-\gamma) \theta\left(x_{t}\right)+\gamma x_{t}$, where the adjustment parameter $\gamma$ is positive but allowed to be greater than unity. The goal of implementation of the adaptive adjustment is to stabilize an economic process $x_{t+1}=\theta\left(x_{t}\right)$ directly through variation of the adjustment parameter $\gamma$, regardless of how the process is formed and what type of expectation is actually assumed in the model. In economics, an adaptive adjustment scheme so defined can be applied to a broader class of discrete economic processes where the variables interested can be adjusted or controlled directly by economic agents, such as in cobweb dynamics, Cournot games, Oligopoly markets, tatonnement price adjustment, tariff games, population control through immigration etc.

Despite the fact that partial adjustment models have been widely utilized in economic analysis, by which an economic variable is purposely adjusted towards its desired value, the idea of stabilizing an economic variable to a desired but a priori unknown equilibrium through adaptive adjustment, to our knowledge, has never been formally addressed. Due to the increasing complexity arising from higher dimensions, the convergence issue is not as trivial as expected to be. As a matter of fact, not all discrete processes can be stabilized through adaptive adjustment in the conventional sense - where the adjustment parameter is restricted between zero and unity.

On the other hand, a rapidly growing interest in complex and chaotic economic dynamics has been witnessed in the last two decades. Although chaos can be a beneficial feature on some rare occasions (Huang, 1995), its undesirable characteristics such as irregularity of orbits and strong sensitivity to initial conditions and perturbations generally lead to detrimental consequences. It is therefore wished that chaos could be suppressed or adjusted so as to force a dynamical economic process to converge to a desired and "stable" equilibrium. Controlling chaos, or more generally, stabilizing a unstable dynamical process, thus has become a fascinating topic recently and various algorithms and methods have been proposed (see Chen and Dong, 1998 and references therein). Being effective in science and engineering, these algorithms are difficult to implement in economics due to the reason that a priori information about the processes variables and internal structure are always demanded. The 
adaptive adjustment mechanism, however, overcomes such limitations and is proved to be an effective stabilizing mechanism for a chaotic process.

The paper is organized as follows. The ensuing section briefly discusses the connection between conventional adaptive expectations and adaptive adjustment mechanisms in a one-dimensional discrete process. Some basic properties are summarized. Section 3 makes a simple generalization - the adaptive parameter is allowed to exceed unity. Such generalization greatly improves the power of the adaptive adjustment mechanism in stabilization. Section 4 turns to higher dimensional processes (multiple variable processes), where uniformly adaptive adjustment is introduced and analyzed, by which all economic variables are adjusted with the same speed. We show that such adjustment succeeds in stabilizing type-I and typeII steady states (the steady states with all eigenvalues either greater than unity or less than unity). The situation where economic variables are adjusted with different speeds (that is, non-uniformly adaptive adjustment) is the main focus of Section 5. Section 6 turns to the controllability issue. Possible areas for further research and concluding remarks are addressed in the last section.

\section{FROM ADAPTIVE EXPECTATIONS TO ADAPTIVE ADJUSTMENT}

In a traditional cobweb model, a perfectly competitive firm must make its output decision one period in advance of the actual sale - such as in agriculture, fishing, forestry, and construction, where the application of production inputs must precede by an appreciable length of time the sale of the output. It is assumed that the firm supplies its output $Q_{t}$ based on the expected price $P_{t}^{e}$, that is, $Q_{t}=S\left(P_{t}^{e}\right)$ and that the actual price $P_{t}$ adjusts to demand so as to clear the market, that is,

$$
D\left(P_{t}\right)=S\left(P_{t}^{e}\right)
$$

where $D\left(P_{t}\right)$ is the market demand function.
Under conventional monotonic assumptions $\left(D^{\prime}(\cdot)<0\right.$ and $\left.S^{\prime}(\cdot)>0\right)$ and so called naive expectations $P_{t}^{e}=P_{t-1}$, the market clearing condition (1) yields the so called quantity dynamics: $Q_{t}=f\left(Q_{t-1}\right)=S\left(D^{-1}\left(Q_{t-1}\right)\right)$.

If an adaptive expectation is adopted instead, that is, $P_{t}^{e}=\alpha P_{t-1}^{e}+(1-\alpha) P_{t-1}$, where $0 \leq \alpha \leq 1$, the quantity dynamics turn turns into $Q_{t}=$ $S\left(\alpha S^{-1}\left(Q_{t-1}\right)+(1-\alpha) D^{-1}\left(Q_{t-1}\right)\right.$.

When $S$ takes a linear form, we then have

$$
\begin{aligned}
Q_{t} & =\alpha Q_{t-1}+(1-\alpha) S\left(D^{-1}\left(Q_{t-1}\right)\right) \\
& =(1-\alpha) f\left(Q_{t-1}\right)+\alpha Q_{t-1}
\end{aligned}
$$

that is, adopting the adaptive expectation rule amounts to adjust adaptively the output $Q_{t}$ directly with the same adjustment speed $\alpha$.

Heiner (1992) explained the rationale for why economic agents would adaptively adjust to new conditions, should they be unable to fully understand the dynamic complexity of the optimal decision over time. As he elaborated: "Given the endogenously implied result, agents should focus their attention on searching for an equilibrium instead of trying to remain optimal at each instant while dynamically adjusting".

Stimulated by the format of (2), we consider an one-dimensional discrete economic process defined by a first order difference equation:

$$
x_{t+1}=f\left(x_{t}\right),
$$

where $f\left(x_{t}\right)$ is a nonlinear function well defined in a domain $I=\left[x_{\min }, x_{\text {max }}\right]$. Here, the function $f$ can be either "single humped" or "multiple humped", either continuous or discontinuous, either smooth or non-smooth (in the sense of $C^{1}$ ), but must intersect the diagonal axis $x_{t+1}=x_{t}$ at least once. That is, there exists at least one $\bar{x}$ such that $f(\bar{x})=\bar{x}$.

By adaptive adjustment mechanism in conventional sense, we means the following modification to the original process (3):

$$
x_{t+1}=\tilde{f}\left(x_{t}\right) \hat{=}(1-\gamma) f\left(x_{t}\right)+\gamma x_{t}
$$




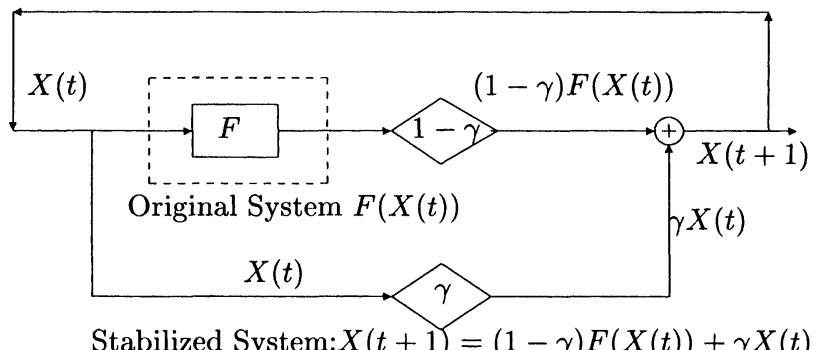

FIGURE 1 Adaptive Adjustment Mechanism.

where the constant $\gamma$ is commonly referred to as adaptive parameter, and is assumed to satisfy the constraint of $0 \leq \gamma<1$. For the convenience of later reference, we call the range between zero and unity as the conventional range.

The conventional adaptive adjustment mechanism resembles closely but not identical to partial adjustment process where an economic variable $x_{t}$ is adjusted gradually to its target $x^{*}$ through the recursive process: $x_{t+1}-x_{t}=\gamma\left(x^{*}-x_{t}\right)$. The convergence to $x^{*}$ is guaranteed as long as $0<\gamma<1$.

In this article, we shall extend our interest beyond the conventional scope of the stability criteria of adaptive expectation and directly explore the possibility of stabilizing a unstable process through adaptive adjustment by (4) focusing on the range of adjustment parameter(s).

The following theorem briefly summarizes some unique properties of adaptive adjustment in the conventional sense.

THEOREM 1 The adjusted process $\tilde{f}\left(x_{t}\right)$ defined by (4) possesses the following mathematical characteristics:

(I) The process $\tilde{f}\left(x_{t}\right)$ preserves the domain of the original process $f\left(x_{t}\right)$.

(II) The processes $f$ and $\tilde{f}$ share exactly the same set of fixed points, that is, for any $\bar{X} \in I=\left[x_{\text {min }}, x_{\text {max }}\right]$, if $f(\bar{x})=\bar{x}$, then $\tilde{f}(\bar{x})=\bar{x}$.

(III) Adaptive adjustment stabilizes the original process in the sense that

$\left|\tilde{f}^{\prime}(x)\right| \leq\left|f^{\prime}(x)\right|$, if $f^{\prime}(x) \geq 1$ or $f^{\prime}(x) \leq 0$, and

$$
1 \geq \tilde{f}^{\prime}(x) \geq f^{\prime}(x), \quad \text { if } \quad 0<f^{\prime}(x)<1,
$$

where $0 \leq \gamma<1$.

(IV) The greater the $\gamma$ value, the greater the stabilizing effect.

Although these properties are straightforward, we still include a proof just for the convenience of later comparison and references.

Proof

(I) Let the interval $I=\left(x_{\min }, x_{\max }\right)$, with $x_{\min }<$ $x_{\max }$, be the domain of the chaotic process $f$, and suppose that $x_{\min }$ and $x_{\max }$ are achieved by $f$ at $x^{l}$ and $x^{h}$, respectively, i.e., $x_{\min }=f\left(x^{l}\right)$ and $x_{\max }=f\left(x^{h}\right)$, with $x_{\min } \leq x^{l} \leq x^{\mathrm{h}} \leq x_{\max }$, then

$$
\begin{aligned}
\tilde{f}\left(x^{l}\right) & =(1-\gamma) f\left(\left(x^{l}\right)+\gamma x^{l}\right. \\
& =(1-\gamma) x_{\min }+\gamma x^{l} \\
& \geq(1-\gamma) x_{\min }+x_{\min }=x_{\min }, \\
\tilde{f}\left(x^{h}\right) & =(1-\gamma) f\left(x^{h}\right)+\gamma x^{h} \\
& =(1-\gamma) x_{\max }+\gamma x^{h} \\
& \leq(1-\gamma) x_{\max }+x_{\max }=x_{\max }
\end{aligned}
$$

that is, $\tilde{f}$ maps $I$ into $I$ itself.

(III) The property is directly concluded from the following identity:

$$
\tilde{f}^{\prime}(x)=(1-\gamma) \tilde{f}^{\prime}(x)+\gamma .
$$


(IV) Taking the partial derivative with respect to $\gamma$ over both sides of (5) gives

$$
\begin{aligned}
\frac{\partial}{\partial \gamma} \tilde{f}^{\prime}(x)= & 1-\tilde{f}(x), \\
& \text { for any } \quad \gamma \in(0,1) \text { and } x \in I .
\end{aligned}
$$

We see that $\left(\partial / \partial_{\gamma}\right) \tilde{f}^{\prime}(x)$ takes a positive value if $f^{\prime}(x)<1$ and negative value if $f^{\prime}(x)<1$.

By intuition, the greater the $\gamma$ value is, the less the original process $f$ is weighted in the adaptive adjustment mechanism.

Q.E.D.

Property III reveals that, under conventional adaptive adjustment, all branches of $f$ with negative slopes are tilted counter-clockwise around the fixed points, while all branches of $f$ with positive slopes that are greater than unity in value are tilted clockwise. Even though the degrees of steepness of those branches with slope between zero and unity are increased slightly, the resulting slopes still stay in the "stable" region (less than unity). And hence, adaptive adjustment effectively "stabilizes" those unstable fixed points while leaving stable fixed points intact. Geometrically, as illustrated by Figure 2, the conventional adaptive adjustment mechanism actually "squeezes" the original process towards its diagonal axis: $x_{n+1}=x_{n}$.

It follows from above the discussion that, for an one-dimensional dynamical process defined by (3), if there exists at least one fixed point $\bar{x}$ such that $f^{\prime}(\bar{x})<0$, then there always exists a constant $\gamma^{*}$ defined by

$$
\gamma^{*} \hat{=} \frac{-1-f^{\prime}(\bar{x})}{1-f^{\prime}(\bar{x})}
$$

such that for all $\gamma \in\left(\gamma^{*}, 1\right]$, the process under adaptive adjustment given by (4) will converge to the stable fixed point. The convergence is guaranteed for all the initial points sufficiently close to this particular fixed point.

It deserves mention that Property II does not hold for periodic orbits (i.e., fixed points of higher order). Although there does exist an one-to-one correspondence between the periodic points of $f$ and those of $\tilde{f}$, the exact locations of these periodic points are actually different. This results from the fact that the solutions to $\bar{x}^{(k)}=f^{k}\left(\bar{x}^{(k)}\right)$ and to $\bar{x}^{(k)}=\tilde{f}^{k}\left(\bar{x}^{(k)}\right)$ are no longer the same if $k$ is greater than one. Although this is acceptable in most applications, there are situations where the original periodic orbits are preferred. In this regard, we
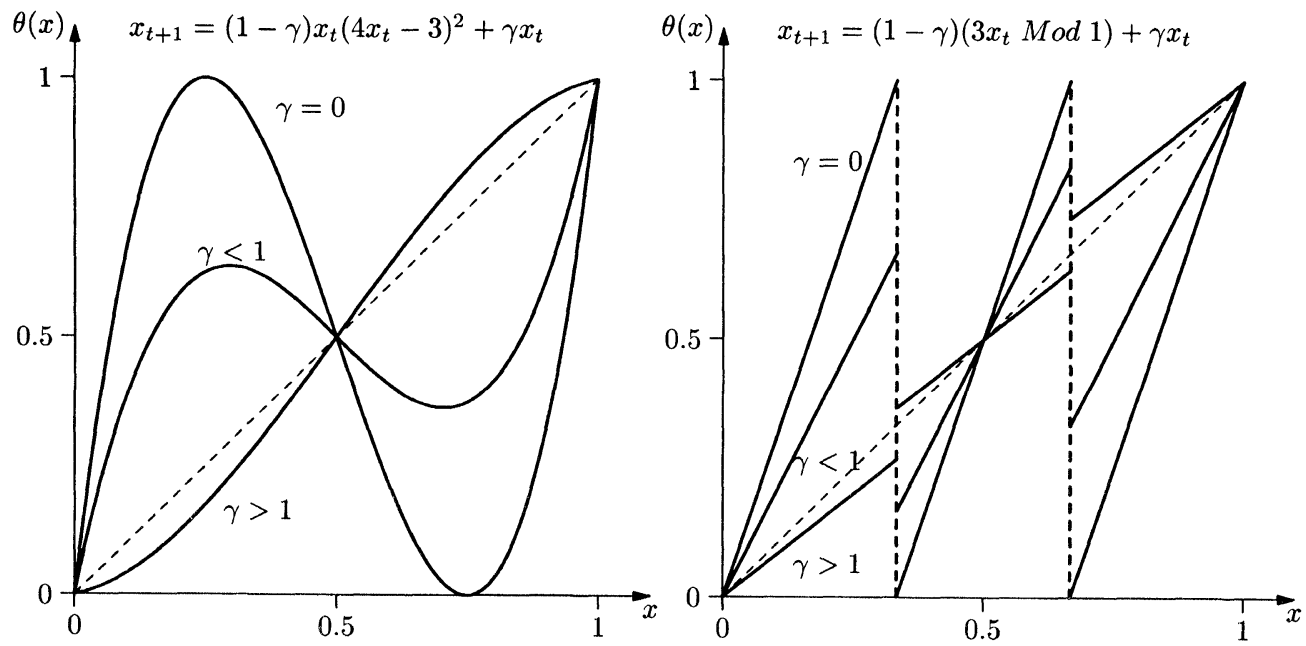

FIGURE 2 Effects of Adaptive Adjustment. 
need to modify the adaptive adjustment mechanism accordingly so to insure the trajectories converge to a desired periodic orbit.

Redefine the adaptive adjustment mechanism as

$$
x_{t+1}=\tilde{f}_{m}\left(x_{t}\right) \hat{=}(1-\gamma) f^{m}\left(x_{t}\right)+\gamma x_{t}
$$

where $f^{m} \hat{=} \underbrace{f \circ f \circ \cdots \circ f}_{m \text { times }}$ denotes $m$ th recurrent process of $f$. By similar arguments, the set of fixed points of $\tilde{f}_{m}$ are identical to the ones of $f^{m}$, which implies that, with a suitable choice of $\gamma$, the adaptive adjustment algorithm defined by (8) can lead to a stable periods- $m$ orbit inherited from $f$.

\section{FROM CONVENTIONAL RANGE TO GENERALIZED RANGE}

Conventional adaptive adjustment mechanism proposed in the last section is inherited from adaptive expectation widely applied in economic analysis and is effective in stabilizing only fixed points with negative derivatives. This limit seriously impairs its effectiveness in application to economic analysis. While it is true that bending and folding are common characteristics for a chaotic process to exhibit perpetual aperiodic phenomena, the existence of a fixed point with a negative derivative may not be guaranteed for processes such as $x_{t+1}=3 x_{t} \bmod 1$, for $x \in[0,1]$ as illustrated in Figure 2b.

Even if there exist such fixed points with negative derivatives, they may be undesirable or of no economic meaning under a particular economic context. We need to have a mechanism that can overcome this limitation so as to ensure the convergence of an adjusted nonlinear process to any desirable fixed point, no matter what sign its derivative may take.

To this end, we generalize adaptive adjustment by extending its adjustment parameter to exceed unity.

Denote $\bar{x}$ as the fixed point of $f$ with slope greater than unity, i.e., $f(\bar{x})>1$, then it follows from identity (5) that $\left|\tilde{f}^{\prime}(\bar{x})\right|<1$ can be easily achieved by extending adaptive parameter $\gamma$ to the range of $\left(1,\left(f^{\prime}(\bar{x})+1\right) /\left(f^{\prime}(\bar{x})-1\right)\right)$.

Such generalization may not have the same economic interpretation as the conventional adaptive expectation, but it is mathematically feasible and practically effective. In order to distinguish from conventional parameter range, we refer to $\gamma>1$ as the generalized range.

To see the effect of adaptive adjustment with $\gamma$ taking values in the generalized range, we recall that $\tilde{f}^{\prime \prime}(x)=(1-\gamma) f^{\prime \prime}(x)$. In contrast to the case with $\gamma<1$, in which $\tilde{f}^{\prime \prime}(x)$ preserves the same sign of $f^{\prime \prime}(x)$ and the net effect is to squeeze the original process toward the diagonal line (under most circumstances), now $\tilde{f}^{\prime}$ has the opposite sign of $f^{\prime}(x)$ in most cases, while $\tilde{f}^{\prime \prime}(x)$ is always opposite to $f^{\prime \prime}(x)$. So the net effect of adaptive adjustment in the generalized range is to reflect the original process against the diagonal line in the phase diagram. These points are well demonstrated in Figures $2 a-2 b$, where thick lines are for the original process, medium lines for the process adjusted in the conventional range, and thin lines for the process adjusted in the generalized range.

Example 1 Consider a cubic process defined by

$$
x_{t+1}=\theta\left(x_{t}\right) \hat{=} x_{t}\left(4 x_{t}-3\right)^{2},
$$

and illustrated in Figure $2 \mathrm{a}$, from which we see that the fixed point $\bar{x}_{2}=(1 / 2)$ is stabilized with conventional AAM with $\gamma=(1 / 2)$, the fixed point at two ends $x_{1}=0$ and $x_{3}=1$, however, can only be stabilized by generalized AAM with $\gamma \in[1$, $\left.\gamma_{\max }\right]$, where $\gamma_{\max }=\left(\left(\theta^{\prime}(0)+1\right) /\left(\theta^{\prime}(0)-1\right)\right)=(5 / 4)$. However, no matter what value $\gamma$ may take, adaptive adjustment always preserve the positions of these fixed points.

Finally, we emphasize that, while Properties II to IV stated in Theorem 1 still hold for the generalization, Property I may not hold true anymore, because the domain for bounded dynamics may be narrowed if $\gamma$ is allowed to exceed unity. 


\section{FROM ONE DIMENSION TO MULTI-DIMENSION}

The principal of adaptive adjustment can be easily applied to multi-dimensional discrete processes, but the analysis turns out to be much more complicated due to the presence of complex eigenvalues and a lack of direct correspondence between eigenvalues and the adjustment parameters.

Consider a $n$-dimensional dynamical process defined by

$$
\mathbf{X}_{t+1}=\mathbf{F}\left(\mathbf{X}_{t}\right),
$$

where $\mathbf{X}_{t}=\left(x_{1 t}, x_{2 t}, \ldots, x_{n t}\right)$, and $\mathbf{F}=\left(f_{1}, f_{2}, \ldots\right.$, $f_{n}$ ), with $f_{i}$ being well defined functions on a domain $I^{n}$.

Definition 1 By Adaptive Adjustment Mechanism (AAM for short), we mean the following adjusted process:

$$
\mathbf{X}_{t+1}=\tilde{\mathbf{F}}_{\Gamma}=(\mathbf{I}-\Gamma) \mathbf{F}\left(\mathbf{X}_{\mathbf{t}}\right)+\Gamma \mathbf{X}_{\mathbf{t}},
$$

where $\Gamma=\operatorname{diag}\left\{\gamma_{1}, \gamma_{2}, \ldots, \gamma_{n}\right\}$ is a diagonal matrix, with $\gamma_{i} \geq 0$, for $i=1,2, \ldots, n$ and is referred to as an adaptive parameter matrix hereafter.

Expressing (10) as $\mathbf{X}_{t+1}=\mathbf{F}\left(\mathbf{X}_{\mathbf{t}}\right)+\Gamma\left(\mathbf{X}_{\mathbf{t}}-\mathbf{F}\left(\mathbf{X}_{\mathbf{t}}\right)\right)$, we see that AAM forces an adjustment whenever the relevant process variables stray away from its previous state. The practical implementation is illustrated in Figure 1.

Let $\overline{\mathbf{X}}$ be the fixed point of (9), that is, $\overline{\mathbf{X}}=\mathbf{F}(\overline{\mathbf{X}})$. It is easy to see that the process $\tilde{\mathbf{F}}_{\Gamma}\left(\mathbf{X}_{t}\right)$ shares exactly the same set of fixed points of $\mathbf{F}$, that is, $\overline{\mathbf{X}}=\tilde{\mathbf{F}}_{\Gamma}(\overline{\mathbf{X}})$, which will be referred to as the generic property for later reference. The other properties stated in Theorem 1, however, can not be similarly satisfied in the multi-dimensional implementation of AAM.

Denote $\mathcal{J}(\overline{\mathbf{X}})$ as the Jacobian matrix of the original process $\mathbf{F}$ evaluated at $\overline{\mathbf{X}}$ with $\left\{\lambda_{1}, \lambda_{2}, \ldots, \lambda_{n}\right\}$ as the $n$ roots of the characteristic equation: i.e.,

$$
|\lambda \mathbf{I}-\mathcal{J}(\overline{\mathbf{X}})|=\prod_{j=1}^{n}\left(\lambda-\lambda_{j}\right)=0,
$$

where $\mathbf{I}$ is a unit matrix.
The stability of a fixed point, $\overline{\mathbf{X}}$, is jointly determined by all the eigenvalues $\left\{\lambda_{j}\right\}$. Let $\left|\lambda_{\max }\right|=\max _{j}\left|\lambda_{j}\right|$. Mathematically, the fixed point $\overline{\mathbf{X}}$ is stable if $\left|\lambda_{\max }\right|<1$.

We are only concerned with the unstable fixed points, that is, the fixed points with $\left|\lambda_{\max }\right| \geq 1$.

Denote a pair of complex conjugates $\lambda_{j}$ and $\bar{\lambda}_{j}$ by

$$
\lambda_{j}=a_{j}+b_{j} \mathbf{i}, \quad \bar{\lambda}_{j}=a_{j}-b_{j} \mathbf{i},
$$

with the modules $\left|\lambda_{j}\right|=\left|\bar{\lambda}_{j}\right|=\sqrt{a_{j}^{2}+b_{j}^{2}}$.

For the convenience of later reference, we classify a unstable fixed point according to the modulus of related eigenvalues as follows:

Definition 2 (Classification of Unstable Fixed Points) Type-I Unstable Fixed Points $a_{j}<1$, for all $j$, i.e., the fixed points with all eigenvalues less than unity in real parts;

Type-II Unstable Fixed Points $a_{j}>1$, for all $j$, i.e., the fixed points with all eigenvalues greater than unity in real parts;

Type-III Unstable Fixed Points $a_{i}>1, a_{j}<1$ for some $i, j$, i.e., the fixed points with some real parts greater than unity, others less than unity in real parts;

Type-IV Unstable Fixed Points there exists at least one $j$ such that either $a_{j}=1$ or $\lambda_{j}=1$, i.e., the fixed points with unity eigenvalues.

The objective of adaptive adjustment is to stabilize a unstable fixed point such that, after introducing an appropriate adaptive parameter matrix $\Gamma=\operatorname{diag}\left\{\gamma_{1}, \gamma_{2}, \ldots, \gamma_{n}\right\}$, all eigenvalues, denoted by $\tilde{\lambda}_{j}, j=1,2, \ldots, n$ become less than unity in modulus. Unfortunately, except for some special situations, there does not exist an one-toone relationship between $\lambda_{j}, \gamma_{j}$ and $\tilde{\lambda}_{j}$ like identity (5) in general, and hence the analysis of the effect of each $\gamma_{j}$ on $\tilde{\lambda}_{j}$ turns out to be extremely difficult in high dimensional cases.

Instead of addressing the general adaptive matrix $\Gamma$ directly, we start with one special case in which a simple relationship analogous to identity (5) can be established. 
This special case is related to the so called uniformly adaptive adjustment,

$$
\Gamma=\{\gamma, \gamma, \ldots, \gamma\}=\gamma \mathbf{I}_{n},
$$

that is, all economic variables are adjusted with same speed:

$$
X_{t+1}=\tilde{F}=(1-\gamma) F\left(X_{\mathbf{t}}\right)+\gamma X_{\mathbf{t}} .
$$

We shall see that the discussion with such simplified process, which is commonly seen in economic modeling where all endogenous variables are adjusted by a single economic agent (tatonnement process, for instance), can provide us invaluable insight into the mechanism of adaptive adjustment in general.

Let $\tilde{\mathcal{J}}(\overline{\mathbf{X}})$ be the Jacobian matrix of the process $\tilde{\mathbf{F}}$ evaluated at $\tilde{\mathbf{X}}$ and $\left\{\tilde{\lambda}_{1}, \tilde{\lambda}_{2}, \ldots, \tilde{\lambda}_{n}\right\}$ be the related eigenvalues, so that

$$
|\lambda \mathbf{I}-\tilde{\mathcal{J}}(\overline{\mathbf{X}})|=\prod_{j=1}^{d}\left(\lambda-\tilde{\lambda}_{j}\right)=0 .
$$

Then we have

THEOREM 2 For each and every fixed point of $F$ and $\tilde{F}$, there exists the following one-to-one correspondence between their eigenvalues:

$$
\tilde{\lambda}_{j}=(1-\gamma) \lambda_{j}+\gamma, \quad j=1,2, \ldots, n .
$$

Proof It follows from (11) that

$$
\tilde{\mathcal{J}}(\overline{\mathbf{X}})=(1-\gamma) \mathcal{J}(\overline{\mathbf{X}})+\gamma \mathbf{I}
$$

The characteristic equation under the process $\tilde{\mathbf{F}}$ is given by

$$
\begin{aligned}
|\tilde{\lambda} \mathbf{I}-\tilde{\mathcal{J}}(\overline{\mathbf{X}})| & =|(\tilde{\lambda}-\gamma) \mathbf{I}-(1-\gamma) \mathcal{J}(\overline{\mathbf{X}})| \\
& =(1-\gamma)^{n}|\hat{\lambda} \mathbf{I}-\mathcal{J}(\overline{\mathbf{X}})| \\
& =(1-\gamma)^{n} \prod_{j=1}^{n}\left(\hat{\lambda}-\lambda_{j}\right),
\end{aligned}
$$

where $\hat{\lambda} \hat{=}((\tilde{\lambda}-\gamma) /(1-\gamma))$, so that $\hat{\lambda}=\lambda_{j}$ would imply identity (13).

Q.E.D.
Theorem 2 and the generic property together enable us to adjust the eigenvalues to become less than unity in modulus by suitable choice of a single adaptive parameter $\gamma$ only.

To get a general picture of the role played by the adjustment parameter $\gamma$, we examine the situation in which a pair of conjugates are presented.

Adopting the same notations introduced before, for an eigenvalues $\lambda_{j}=a_{j}+b_{j} \mathbf{i}$ related to the original process $\mathbf{F}$, its counterparts from $\tilde{\mathbf{F}}$ is

$$
\begin{aligned}
\tilde{\lambda}_{j}= & (1-\gamma)\left(a_{j}+b_{j} \mathbf{i}\right)+\gamma=\left[(1-\gamma) a_{j}+\gamma\right] \\
& +(1-\gamma) b_{j} \mathbf{i} .
\end{aligned}
$$

The modulus is given by $\left|\tilde{\lambda}_{j}\right|=\sqrt{H_{j}(\gamma)}$, where

$$
H_{j}(\gamma) \hat{=}\left((1-\gamma) a_{j}+\gamma\right)^{2}+(1-\gamma)^{2} b_{j}^{2}
$$

Let $\bar{\gamma}_{j}$ be the critical adaptive parameter such that $H_{j}\left(\bar{\gamma}_{j}\right)=1$. Solving from (15), we have

$$
\bar{\gamma}_{j}=1+\frac{2\left(a_{j}-1\right)}{\left(a_{j}-1\right)^{2}+b_{j}^{2}},
$$

and $H_{j}^{\prime}(\bar{\gamma})=2\left(a_{j}-1\right)$. Therefore, $\quad \bar{\gamma}_{j} \lessgtr 1$ and $H_{j}^{\prime}\left(\bar{\gamma}_{j}\right) \lessgtr 0$ if and only if $a_{j} \lessgtr 1$.

For the special cases $\gamma=0$ (without AAM) and $\gamma=1$ (no effect of the original process), there exist the following identities and inequalities:

$$
\begin{aligned}
& H_{j}(0)=a_{j}^{2}+b_{j}^{2}>0, \\
& H_{j}^{\prime}(0)=2\left(a_{j}-H_{j}(0)\right), \\
& H_{j}(1)=1, \\
& H_{j}^{\prime}(1)=2\left(1-a_{j}\right) \lessgtr 0 \quad \text { if } \quad a_{j} \lessgtr 0 .
\end{aligned}
$$

Also note that

$$
H_{j}^{\prime \prime}(\gamma)=2\left(\left(1-a_{j}\right)^{2}+b_{j}^{2}\right)>0 .
$$

These relationships enable us to explore the way $H_{j}(\gamma)$ is changed with $\gamma$ in term of the nature of $H_{j}(0)$ :

Case $A \quad H_{j}(0)>1$ (and hence $\left|\lambda_{j}\right|>1$ ): If $a_{j}<1$, we have $H_{j}^{\prime}(0)<0$ and $H_{j}^{\prime}(1)>0$. The identity 
$H_{j}(1)=1$ implies that there always exists a $\bar{\gamma}_{j}$ such that $H_{j}\left(\gamma_{j}\right)<1$ for all $\gamma \in\left(\bar{\gamma}_{j}, 1\right)$. However, when $\gamma>1, H_{j}(\gamma)$ will resume to exceed unity.

If $a_{j}>1$, both $H_{j}^{\prime}(0)<0$ and $H_{j}^{\prime}(1)<0$ hold true. The convex property of $H_{j}$ reveals that $H_{j}$ is decreasing along the increase of $\gamma$ from 0 to 1 (but never to the extent that it is less than unity). Therefore, there always exists a $\bar{\gamma}_{j}$ such that $H(\gamma)<1$ for all $\gamma \in\left(1, \bar{\gamma}_{j}\right)$. But when $\gamma>\bar{\gamma}, H_{j}(\gamma)$ starts to exceed unity again.

If $a_{j}=1$, although $H_{j}^{\prime}(\gamma)<0$ holds for $\gamma<1$, $H_{j}(\gamma)$ is always greater than unity.

The above analysis is illustrated in Figure 3a with the omission of subscript $j$, where $H(0) \equiv 4$ is assumed, and $H(\gamma)$ is plotted against $\gamma$. We see, no matter what $a$ is, uniformly adaptive adjustment with $\gamma<1$ always helps in reducing the magnitude of the modules. It also observed that the modulus of an imaginary eigenvalue $(a=0, b>1)$ can only be reduced by a $\gamma$ that is less than unity.

Case $B \quad H_{j}(0)<1$ (and hence $\left|\lambda_{j}\right|<1$ ): This case exists only when $a_{j}<1$. Since $H_{j}^{\prime}(0)=$ $2\left(a_{j}-H_{j}(0)\right)$, introducing a $\gamma$ that is less than unity may decrease or increase the eigenvalue at the beginning, but finally increased again until $H_{j}(1)=1$. Hence, when $\gamma \in(0,1), H_{j}(\gamma)$ will never exceed unity so that the stability of a fixed point is preserved. To the contrary, when $\gamma>1, H_{j}(\gamma)$ will become greater than unity so as to destabilize a stable fixed point. Case B is illustrated in Figure 3b, where $H(0) \equiv 0.6$ is set.

When the original eigenvalue is real $\left(\lambda_{j}=a_{j}\right)$, identity (16) is simplified to

$$
\bar{\gamma}_{j}=\frac{\left(\lambda_{j}+1\right)}{\lambda_{j}-1}
$$

which is the multi-dimensional analogue of (7). The relationship between $\lambda$ and $\tilde{\lambda}$ with respect to $\gamma$ is demonstrated in Figure 4.

It follows directly from the above analysis that:

Theorem 3 For a n-dimensional dynamical process $X_{t+1}=F\left(X_{t}\right)$, a unstable fixed point $\bar{X}$ can be stabilized through uniformly adaptive adjustment defined by (11) if and only $\bar{X}$ is either a type-I fixed point $\left(a_{j}<1\right.$ for all $\left.j=1,2, \ldots, n\right)$ or a type-II fixed point $\left(a_{j}>1\right.$ for all $\left.j=1,2, \ldots, n\right)$.

Proof Let $a_{j}$ and $b_{j}$ be the real part of eigenvalues $\lambda_{j}, j=1,2, \ldots, n$, associated with a fixed point $\bar{X}$. respectively, and define $\bar{\gamma}_{j}=1+\left(\left(2\left(a_{j}-1\right)\right) /\right.$ $\left.\left(\left(a_{j}-1\right)^{2}+b_{j}^{2}\right)\right)$, for $j=1,2, \ldots, n$. Denote $\bar{\gamma}_{\min }=\min \left\{\bar{\gamma}_{1}, \bar{\gamma}_{2}, \ldots, \bar{\gamma}_{n}\right\} \quad$ and $\quad \bar{\gamma}_{\max }=\max \left\{\bar{\gamma}_{1}\right.$, $\left.\bar{\gamma}_{2}, \ldots, \bar{\gamma}_{n}\right\}$.

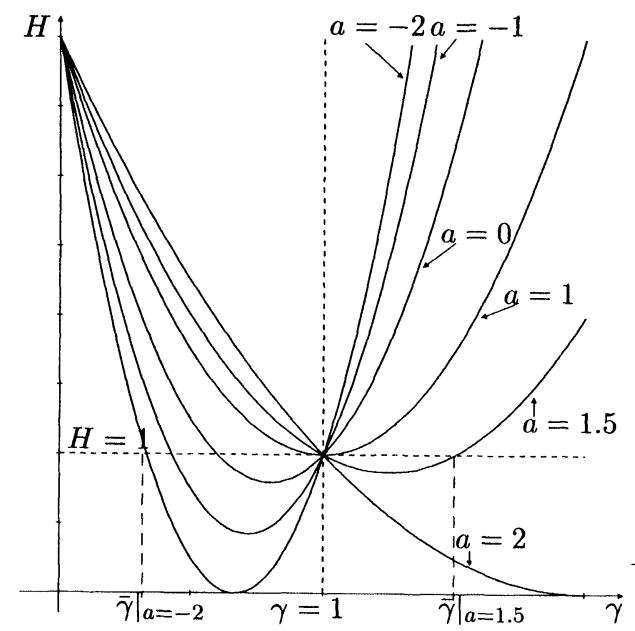

(a)Unstable fixed Orbit

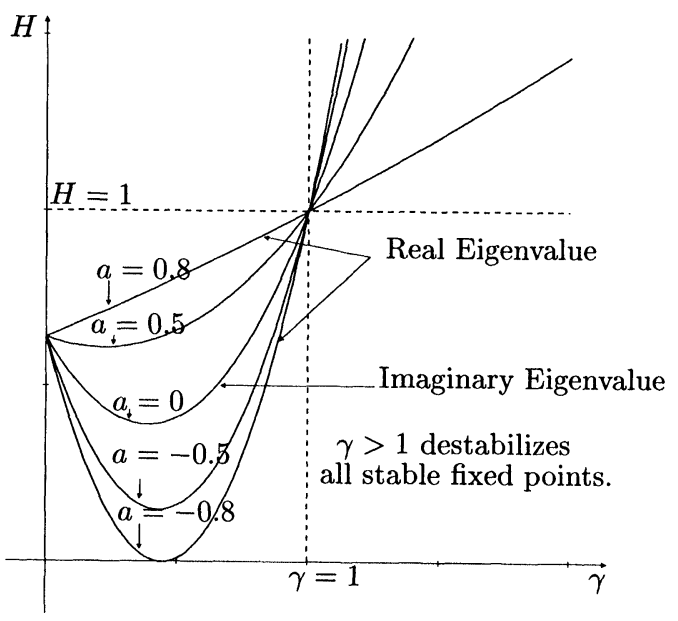

(b)Stable fixed Orbit

FIGURE 3 Effects of $\gamma$. 


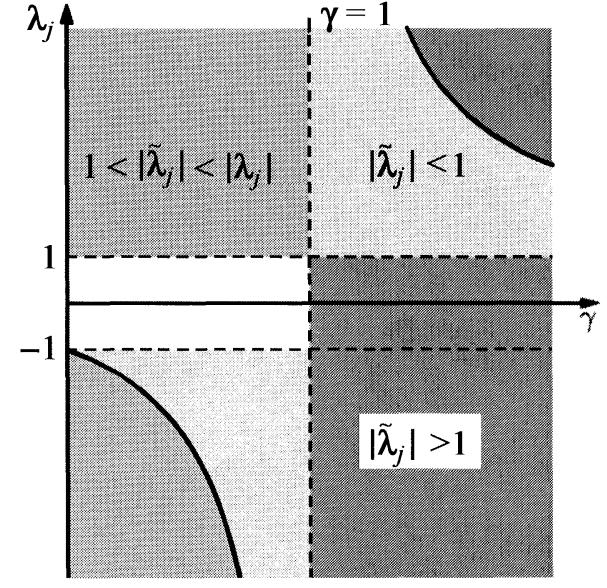

FIGURE 4 Effects of $\gamma$-Real Eigenvalue.

When $\mathbf{X}$ is a type-I fixed point, that is, $a_{j}<1$ for all $j=1,2, \ldots, n$, (but some $a_{j}<-1$ ), from the reasoning above, for every $j$, there exists an interval $\left(\bar{\gamma}_{j}, 1\right)$ such that the eigenvalue resulting from AAM will be less than unity in modulus (i.e., $\left.\left|\tilde{\lambda}_{j}\right|<1\right)$, if $\gamma \in\left(\bar{\gamma}_{j}, 1\right)$. Therefore, if $\gamma \in\left(\bar{\gamma}_{\max }, 1\right)$, $\left|\tilde{\lambda}_{j}\right|<1$ for all $j$.

Similarly, if $\overline{\mathbf{X}}$ is a type-II fixed point, that is, $a_{j}>1$ for all $j=1,2, \ldots, n$, stability condition $\left|\tilde{\lambda}_{j}\right|<1$ for all $j$ is guaranteed when the adaptive parameter $\gamma$ is take in the interval $\left(1, \bar{\gamma}_{\min }\right)$.

Q.E.D.

Example 2 Consider the Hennon process $\mathbf{X}_{t+1}=$ $\theta\left(\mathbf{X}_{t}\right)$, defined by

$$
\left.\begin{array}{l}
x_{1 t+1}=\frac{7}{5}+\frac{3}{10} x_{2 t}-x_{1 t}^{2} \\
x_{2 t+1}=x_{1 t}
\end{array}\right\}
$$

This is a famous chaotic process with a strange attractor. There are two fixed points: $\overline{\mathbf{X}}_{1} \approx$ $(0.8839,0.8839)$ with eigenvalues $\left\{\lambda_{1}^{(1)}, \lambda_{2}^{(1)}\right\}=$ $\{0.156,-1.924\}$, and $\overline{\mathbf{X}}_{2} \approx(-1.5839,-1.5839)$ with eigenvalues $\left\{\lambda_{1}^{(2)}, \lambda_{2}^{(2)}\right\}=\{3.26,-0.92\}$, respectively. Apparently, $\overline{\mathbf{X}}_{1}$ can be stabilized through uniformly adaptive adjustment since both eigenvalues are less than unity.

$$
\bar{\gamma}_{1}^{(1)}=\frac{\lambda_{1}^{(1)}+1}{\lambda_{1}^{(1)}-1}=-1.3697,
$$

$$
\bar{\gamma}_{2}^{(1)}=\frac{\lambda_{2}^{(1)}+1}{\lambda_{2}^{(1)}-1}=0.31601 .
$$

So it would be expected that the adjusted process

$$
\left.\begin{array}{l}
x_{1 t+1}=(1-\gamma)\left[\frac{7}{5}+\frac{3}{10} x_{2 t}-x_{1 t}^{2}\right]+\gamma x_{1 t} \\
x_{2 t+1}=(1-\gamma) x_{1 t}+\gamma x_{2 t}
\end{array}\right\},
$$

will converge to the fixed point $\overline{\mathbf{X}}_{1} \approx$ $(0.8839,0.8839)$ when $\gamma \in(0.31601,1)$.

Figure 5a shows the bifurcation diagram of $x_{t}$ against the adaptive parameter $\gamma$ after discarding first 300 iterations. Along with the increasing of $\gamma$ the dynamics changes from pure chaos to multiple periodic points, and finally convergance to the stable fixed point $\overline{\mathbf{X}}_{1}$ when $\gamma>0.3$.

To have a better idea of the effectiveness of uniformly adaptive adjustment, two numerical simulations are overlapped together in Figure $5 b$ for the cases of $\gamma=0.2$ and $\gamma=0.4$, respectively. With these two adaptive parameters, the process rapidly converges to a periodic- 2 orbits and the fixed point $\overline{\mathbf{X}}_{1}$, respectively. But it should be emphasized that, while the fixed point converged to, under $\gamma=0.4$ is "generic", the periodic- 2 orbits converged to under $\gamma=0.2$, however, is not inherited from the Hennon process.

Example 3 Oligopolistic Competition (Theocharis (1960)) Consider a market with $n$ oligopolistic firms producing a homogeneous output, and with a linear market demand curve $p_{t}=a-b \sum_{i=1}^{n} x_{i t}$, where $a>0, b>0$ and $x_{i t}$ is the actual output of firm $i$ at time $t$. We also assume linear cost curves $C_{i t}$ for each firm, $C_{i t}=C_{i}+c_{i} x_{i t}$.

Given the assumption that each firm has an ex ante market price expectation based on the belief that the other firms' outputs will remain unchanged, namely

$$
p_{t+1}^{i}=a-b\left(x_{i t+1}+\sum_{j \neq i}^{n} x_{j t}\right)
$$



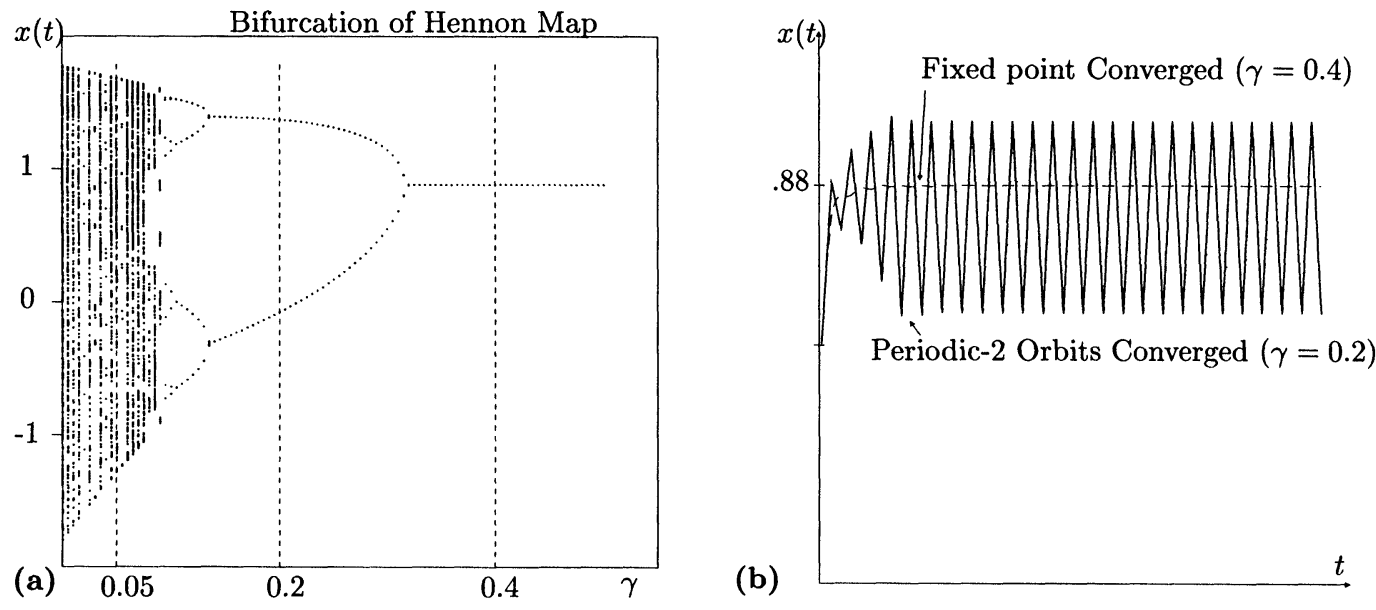

FIGURE 5 (a) Bifurcation Diagram of $\gamma$; (b) Stabilized Orbits under AAM.

on the basis of which firm $i$ determines its output $x_{i t+1}$, aiming at maximizing its expected profit

$$
\begin{aligned}
\pi_{t+1}^{i} & =p_{t+1}^{i} x_{i t+1}-C_{i t+1} \\
& =a x_{i t+1}-b x_{i t+1}^{2}-b x_{i t+1} \sum_{j \neq i}^{n} x_{j t}-\left(C_{i}+c_{i} x_{i t+1}\right)
\end{aligned}
$$

The first order condition gives linear Cournot reaction functions:

$$
x_{i t+1}=\frac{a-c_{i}}{2 b}-\frac{1}{2} \sum_{j \neq i}^{n} x_{j t}, \quad \text { for } i=1,2, \ldots, n .
$$

At an equilibrium, the Jacobian matrix is a constant matrix given by

$$
J=\left(\begin{array}{cccc}
0 & -\frac{1}{2} & \ldots & -\frac{1}{2} \\
\cdots & \cdots & \ldots & \cdots \\
-\frac{1}{2} & 0 & -\frac{1}{2} \cdots & -\frac{1}{2} \\
\cdots & \cdots & \cdots & \cdots \\
-\frac{1}{2} & -\frac{1}{2} & \ldots & 0
\end{array}\right)
$$

It can be verified that the eigenvalue of $J$ is $\lambda_{1}=(-(n-1) / 2)$ and $\lambda_{i}=(1 / 2)$, for $i=2,3, \ldots, n$. Therefore, the process is unstable when $n \geq 3$.

Since all eigenvalues are less than unity, the process can be stabilized through uniformly adaptive adjustment, which can be shown to be identical to the situations that all firms take adaptive expectations with the same weight.

The critical parameter $\bar{\gamma}$ is equal to $\bar{\gamma}=\left(\left(\lambda_{1}+1\right) /\left(\lambda_{1}-1\right)\right)=((n-3) /(n+1))$, that is, the market is stable if all firms take the same adaptive expectation:

$$
p_{t+1}^{i}=\left(1-\gamma^{*}\right)\left(a-b\left(x_{i t+1}+\sum_{j \neq i}^{n} x_{j t}\right)\right)+\gamma^{*} p t
$$

where $\gamma^{*} \in(((n-3) /(n+1)), 1)$, which results in an adaptive adjustment model:

$$
x_{i t+1}=(1-\gamma)\left(\frac{a-c_{i}}{2 b}-\frac{1}{2} \sum_{j \neq i}^{n} x_{j t}\right)+\gamma x_{i t} .
$$

However, in Example 3, it is unrealistic to assume that all firms take on exactly the same adjustment parameter $\gamma$, therefore, we assume that nonuniform adjustment is taken. That is, each firm decides its own adjustment speed, $\gamma_{j}$ so that

$$
x_{i t+1}=\left(1-\gamma_{j}\right)\left(\frac{a-c_{i}}{2 b}-\frac{1}{2} \sum_{j \neq i}^{n} x_{j t}\right)+\gamma_{j} x_{i t} .
$$

The stability of the equilibrium is now determined by the dominant eigenvalues of the Jacobian 
matrix given by

$$
\begin{aligned}
& \tilde{J}= \\
& \left(\begin{array}{cccc}
\gamma_{1} & -\left(1-\gamma_{1}\right) / 2 & \cdots & -\left(1-\gamma_{1}\right) / 2 \\
-\left(1-\gamma_{2}\right) / 2 & \gamma_{2} & -\left(1-\gamma_{2}\right) / 2 \cdots & -\left(1-\gamma_{2}\right) / 2 \\
\ldots & \cdots & \cdots & \cdots \\
-\left(1-\gamma_{n}\right) / 2 & -\left(1-\gamma_{n}\right) / 2 & \cdots & \gamma_{n}
\end{array}\right) .
\end{aligned}
$$

Since the demand function and cost functions are all continuous, so are the reactions functions under AAM given by (20) and the Jacobian matrix $\tilde{J}$. If the dominant eigenvalue of $\tilde{J}$ is less than unity in modulus when all firms adopt the same adaptive weight $\gamma^{*}, \quad \gamma^{*} \in(((n-3) /(n+1)), 1)$, which is a special case of non-uniformly adjustment, we would have no doubt in expecting that, when $\gamma_{j}^{\prime}$ s are sufficient close to $\gamma^{*}$, the dominant eigenvalue of $\tilde{J}$ is still guaranteed to be less than unity in modulus.

\section{FROM UNIFORMLY AAM TO NON-UNIFORMLY AAM}

Adaptive adjustment mechanism with general adaptive matrix may be more realistic in economics where different variables are adjusted by different economic agents with different adjustment speeds. In practice, there prevail the situations where only a part of economic variables are adjustable.

We have concluded that the uniformly adaptive adjustment fails in stabilizing type-III and IV fixed points. We then expect that an AAM with a nonuniformly adaptive matrix $\Gamma$ defined in (10) may overcome such limitations.

By intuition, it seems to be possible to stabilize any type of fixed point by a suitable adaptive parameter matrix $\Gamma$, with some $\gamma_{j}^{\prime}$ s in the conventional range, others in the generalized range. Formally, it is questioned that, for a given nonlinear process (9), if its fixed points are of type-III or type IV, whether there always exists an adaptive parameter matrix $\Gamma=\operatorname{diag}\left\{\gamma_{1}\right.$, $\left.\gamma_{2}, \ldots, \gamma_{n}\right\}$, with at least one $i$ and $j$ such that $\gamma_{i} \neq \gamma_{j}$, such that the adjusted process (10) is stabilized at the same fixed point. The answer is unfortunately negative.

Mathematically, a simple relationship between the original eigenvalues and new eigenvalues analogous to identity (13) can be obtained only for some special situations such as recursive processes (to be discussed in the sequel). Now that all economic variables are dependent on each other, on one hand, stability may be easily achieved by adaptively adjusting only part of the variables. On the other hand, if each economic agent reacts to the unstable dynamics in different ways, each and every aims at stabilizing its related variable only, the overall result could become totally erratic, should no coordination be taken.

To exemplify the above remarks, we start with the examination of a 2-dimensional discrete process.

Let $\mathcal{J}(\overline{\mathbf{X}})$ be the Jacobian matrix associated with a fixed point $\overline{\mathbf{X}}$ of some two-dimensional process:

$$
\mathcal{J}(\bar{X})=\left(\begin{array}{ll}
a & b \\
c & d
\end{array}\right)
$$

Denote

$$
\begin{aligned}
& \mathcal{T}=a+d=\text { trace of } \mathcal{J} \\
& \mathcal{D}=a d-b c=\text { determinant of } \mathcal{J}, \\
& \mathcal{H}=\mathcal{T}^{2}-4 \mathcal{D}
\end{aligned}
$$

then eigenvalues of $J(\overline{\mathbf{X}})$ can be expressed in term of these invariants, as follows

$$
\lambda_{1.2}=\frac{1}{2}(\mathcal{T} \pm \sqrt{\mathcal{H}})=\frac{1}{2}\left(\mathcal{T} \pm \sqrt{\mathcal{T}^{2}-4 \mathcal{D}}\right)
$$

The stability regime and distribution of unstable fixed points can be depicted in a $(\mathcal{T}, \mathcal{D})$ plane, which is sketcheen in Figure 6.

It is shown that a type-IV fixed point is represented by the divergence bifurcation boundary $\mathcal{T}-\mathcal{D}=1$. While a type-III fixed point $\left(\lambda_{1}>1, \lambda_{2}<1\right)$ occurs only under two situations:

(i) $\mathcal{D}<1$ and $\mathcal{T}-\mathcal{D}>1$; and

(ii) $\mathcal{D}>1, \mathcal{T}-\mathcal{D}<1$, but $\mathcal{H}>0$. 


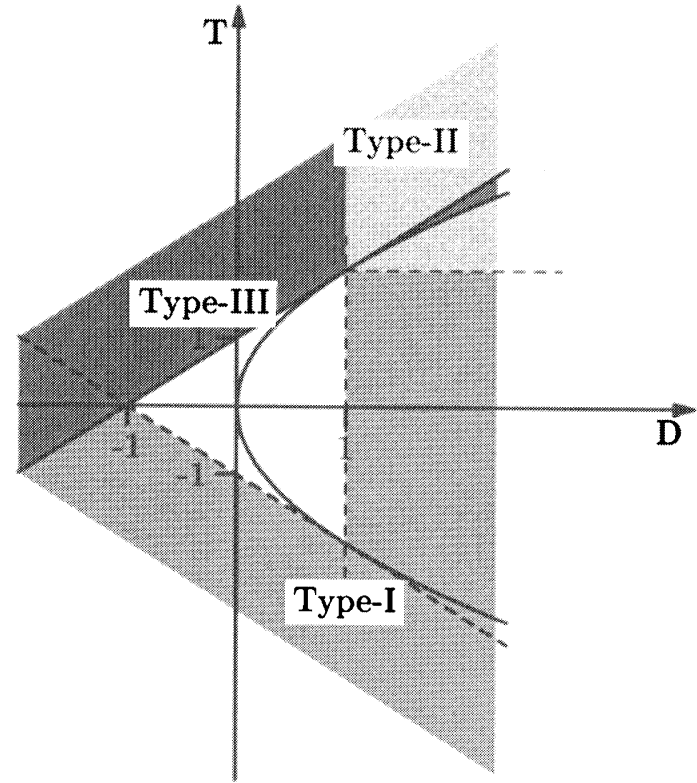

FIGURE 6 Distribution of fixed points.

With adaptive adjustment of $\Gamma=\operatorname{diag}\left\{\gamma_{1}, \gamma_{2}\right\}$, the Jacobian matrix becomes

$$
\tilde{\mathcal{J}}(\overline{\mathbf{X}})=\left(\begin{array}{cc}
\left(1-\gamma_{1}\right) a+\gamma_{1} & \left(1-\gamma_{1}\right) b \\
\left(1-\gamma_{2}\right) c & \left(1-\gamma_{2}\right) d+\gamma_{2}
\end{array}\right),
$$

which gives the eigenvalues $\tilde{\lambda}_{1,2}$ pair as $\tilde{\lambda}_{1,2}=$ $(1 / 2)\left(\tilde{\mathcal{T}} \pm \sqrt{\tilde{\mathcal{T}}^{2}-4 \tilde{\mathcal{D}}}\right)$ where

$$
\tilde{\mathcal{T}}=\mathcal{T}+\gamma_{1}(1-a)+\gamma_{2}(1-d),
$$

and

$\tilde{\mathcal{D}}=\left(1-\gamma_{1}\right)\left(1-\gamma_{2}\right) \mathcal{D}+(1-\mathcal{T}) \gamma_{1} \gamma_{2}+a \gamma_{2}+\gamma_{1} d$.

We see that, even for a 2-dimensional process, the relationship between adjustment parameters $\gamma_{1,2}$, original eigenvalues $\lambda_{1,2}$ and new eigenvalues $\tilde{\lambda}_{1,2}$ becomes very complicated.

It is easy to verify that the simple relationship

$$
\tilde{\lambda}_{j}=\left(1-\gamma_{j}\right) \lambda_{j}+\gamma_{j}, \quad j=1,2
$$

exists if and only if one of the following situations occurs: (i) $\gamma_{1}=\gamma_{2}$, i.e., uniformly adjustment; and (ii) $b \alpha=0$, i.e., recursive processes.
In general, when $\gamma_{1} \neq \gamma_{2}$, each eigenvalue is affected by both adjustment parameters symmetrically, the interaction of these adjustments makes the comparative statistic analysis of overall effects become quite difficult.

Now that uniformly adaptive adjustment, which is a special case of non-uniformly AAM, can stabilize both the type I and type II fixed points, by the continuity argument, we can assure the existence of a non-uniformly adjustment parameter matrix $\Gamma=\operatorname{diag}\left\{\gamma_{1}, \gamma_{2}, \ldots, \gamma_{n}\right\}$ (with at least a pair $(i, j)$ such that $\left.\gamma_{i} \neq \gamma_{j}\right)$ that can stabilize type I and type II fixed points. A type-IV fixed point can only occur when process parameters take some critical values (bifurcation values) and hence is liable to change into either a type-I or a type II fixed point, we shall not discuss.

Then what remain unsolved is type III fixed point, that is, the fixed point with part of the eigenvalues are greater than or equal to unity but the rest are less than unity. Several issues need to be resolved.

At first, even though we have shown that a typeIII fixed point can not be stabilized through uniformly adaptive adjustment, we are still not sure whether they can be stabilized through a combination of adaptive parameters that are not identical but all in the same range (either in conventional range or generalized range). An "impossibility" is shown for a 2-dimensional process.

Actually, for the Jacobian matrices (21) and (22), we have

$$
\tilde{\mathcal{T}}-\tilde{\mathcal{D}}=\left(1-\gamma_{1}\right)\left(1-\gamma_{2}\right)(\mathcal{T}-\mathcal{D}-1)+1,
$$

which implies

(1) A type-IV fixed point can not be stabilized by any $\left(\gamma_{1}, \gamma_{2}\right)$, owing to the fact that $\tilde{\mathcal{T}}-\tilde{\mathcal{D}}=1$ if $\mathcal{T}-\mathcal{D}=1$.

(2) A type-III fixed point with $\mathcal{D}<1$ and $\mathcal{T}$ $\mathcal{D}>1$ can only be stabilized through a combination of $\left(\gamma_{1}, \gamma_{2}\right)$ satisfying the inequality $\left(1-\gamma_{1}\right)\left(1-\gamma_{2}\right)<0$, that is, one takes values in the conventional range, the other takes value in the generalized range. 
Secondly, does there exist any special form processes that AAM always works-not only for type-I or type-II fixed points, but also for type-III fixed points? The answer is definitely "Yes" One of such processes is the recursive process that has been widely applied in economic analysis.

A nonlinear process $\mathbf{F}(\mathbf{X})=\left\{f_{1}(\mathbf{X}), f_{2}(\mathbf{X}), \ldots\right.$, $\left.f_{n}(\mathbf{X})\right\}$, with $\mathbf{X}=\left(x_{1}, x_{2}, \ldots, x_{n}\right)$, is recursive if $f_{i}$ depends only on the first $i$ variables, that is,

$$
\left.\begin{array}{l}
x_{1 t+1}=f_{1}\left(x_{1 t}\right) \\
x_{2 t+1}=f_{2}\left(x_{1 t}, x_{2 t}\right) \\
\ldots \ldots \\
x_{k t+1}=f_{k}\left(x_{1 t}, x_{2 t}, \ldots, x_{k t}\right) \\
\ldots \ldots \\
x_{n t+1}=f_{n}\left(x_{1 t}, x_{2 t}, \ldots, x_{n t}\right)
\end{array}\right\} .
$$

THEOREM 4(Recursive Systems) For a n-dimensional recursive process defined by (23), if $\left.\left(d f_{i} / d x_{i}\right)\right|_{x=\bar{x}} \neq 1$, for $i=1,2, \ldots, n$, then there always exists an adaptive parameter matrix $\Gamma=$ diag $\left\{\gamma_{1}, \gamma_{2}, \ldots, \gamma_{n}\right\}$ such that the adjusted process

$$
\mathbf{X}_{t+1}=\tilde{\mathbf{F}}_{\Gamma}=(\mathbf{I}-\Gamma) \mathbf{F}\left(\mathbf{X}_{\mathbf{t}}\right)+\Gamma \mathbf{X}_{\mathbf{t}},
$$

can be stabilized to its generic fixed point $\overline{\mathbf{X}}$.

Proof If $\mathbf{F}$ is recursive, then at the fixed point $\bar{X}$, its Jacobian matrix is a upper or lower triangular matrix. Following the definition of (24),

$$
\mathcal{J}(\overline{\mathbf{X}})=\left(\begin{array}{cccc}
\frac{d f_{1}}{d x_{1}} & 0 & \cdots & 0 \\
\frac{d f_{2}}{d x_{1}} & \frac{d f_{2}}{d x_{2}} & \cdots & 0 \\
\vdots & \vdots & \ddots & \vdots \\
\frac{d f_{n}}{d x_{1}} & \frac{d f_{n}}{d x_{2}} & \cdots & \frac{d f_{n}}{d x_{n}}
\end{array}\right)_{\mathbf{X}=\overline{\mathbf{X}}}
$$

with eigenvalues $\lambda_{i}=\left(d f_{i} / d x_{i}\right), i=1,2, \ldots, n$. At the same fixed point, the Jacobian matrix for adjusted process (24) becomes

$$
\begin{aligned}
& \tilde{\mathcal{J}}(\tilde{\mathbf{X}})= \\
& \left(\begin{array}{cccc}
\left(1-\gamma_{1}\right) \frac{d f_{1}}{d x_{1}}+\gamma_{1} & 0 & \cdots & 0 \\
\left(1-\gamma_{2}\right) \frac{d f_{2}}{d x_{1}} & \left(1-\gamma_{2}\right) \frac{d f_{2}}{d x_{2}}+\gamma_{2} & \cdots & 0 \\
\vdots & \vdots & \ddots & \vdots \\
\left(1-\gamma_{n}\right) \frac{d f_{n}}{d x_{1}} & \left(1-\gamma_{n}\right) \frac{d f_{n}}{d x_{2}} & \cdots & \left(1-\gamma_{n}\right) \frac{d f_{n}}{d x_{n}}+\gamma_{n}
\end{array}\right)_{X=\bar{X}},
\end{aligned}
$$

which gives rise to the eigenvalues:

$$
\tilde{\lambda}_{i}=\left.\left(1-\gamma_{i}\right) \frac{d f_{i}}{d x_{i}}\right|_{X=\bar{X}}+\gamma_{i}, \quad i=1,2, \ldots, n
$$

It follows from the discussion in previous sections, if $\left.\left(d f_{i} / d x_{i}\right)\right|_{X=\bar{X}} \neq 1$, that is, the fixed point is not of type-IV, there always exists a $\gamma_{i}>0$ such that $\left|\tilde{\lambda}_{i}\right|<1$, for all $i=1,2, \ldots, n$. $\quad$ Q.E.D.

Theorem 4 serves both as an example that a type-III fixed point can be stabilized through nonuniformly adaptive adjustment and as an example that a type-IV fixed point that can not be stabilized through AAM.

\section{FROM JOINTLY AAM TO INDIVIDUALLY AAM}

The last issue deserving our attention is the controllability. As we have commented before, if a multiple-dimensional process is not symmetrical, the effect of each adaptive parameter $\gamma_{i}$, $i=1,2, \ldots, n$, on the stability will be different. There exist situations that some of adaptive parameters are dispensable, that is, the process can still be stabilized if these variables are not adjusted $\left(\gamma_{i}=0\right)$. On the other hand, there are some critical adjustment parameters are indispensable, that is, the stability can not be achieved if any one of them takes zero value. This point can be clearly illustrated through the following three examples. 
Example 4 Part of adjustment parameters are indispensable: For a two dimensional process, if its Jacobian at the fixed point is given by

$$
\mathcal{J}=\left(\begin{array}{cc}
\lambda_{1}+\lambda_{2} & -\lambda_{1} \lambda_{2} \\
1 & 0
\end{array}\right),
$$

then its counterpart from adaptive adjustment

$$
\tilde{\mathcal{J}}=\left(\begin{array}{cc}
\left(1-\gamma_{1}\right)\left(\lambda_{1}+\lambda_{2}\right)+\gamma_{1} & -\left(1-\gamma_{1}\right) \lambda_{1} \lambda_{2} \\
1-\gamma_{2} & 0
\end{array}\right),
$$

will produce $\tilde{\lambda}_{1.2}=(1 / 2)\left(\tilde{\mathcal{T}} \pm \sqrt{\tilde{\mathcal{T}}^{2}-4 \tilde{\mathcal{D}}}\right)$, where

$$
\begin{aligned}
& \tilde{\mathcal{T}}=\gamma_{1}+\left(1-\gamma_{1}\right)\left(\lambda_{1}+\lambda_{2}\right), \\
& \tilde{\mathcal{D}}=\left(1-\gamma_{1}\right)\left(1-\gamma_{2}\right) \lambda_{1} \lambda_{2} .
\end{aligned}
$$

In this case, the adjustment parameter $\gamma_{2}$ has no effect on the real part, so there exist some cases that the dynamics can not be controlled by $\gamma_{2}$ alone.

In fact, if $\gamma_{1}=0$, we have $\tilde{\lambda}_{1,2}=$ $(1 / 2)\left(\left(\lambda_{1}+\lambda_{2}\right) \pm \sqrt{\left.\left(\lambda_{1}+\lambda_{2}\right)^{2}+4 \lambda_{1} \lambda_{2} \gamma_{2}\right)}\right.$. Either $\left|\lambda_{1}+\lambda_{2}\right|>2$, or $\lambda_{1} \lambda_{2}>0, \gamma_{2}$ will become ineffective. Therefore, $\gamma_{1}$ is indispensable.

Example 5 All adjustment parameters are indispensable (Muth, 1961): Consider an isolated market with output lags. Current demand for consumption purposes $C_{t}$ is assumed to depend on current price $p_{t}$, while current production $Q_{t}$, due to the output lag, depends on the price $p_{t}^{e}$ that was expected to hold in the current period. It is also assumed that the commodity is non-perishable, so that inventories $I_{t}$ of it can exist, and are in fact held for speculative purposes, i.e., to profit from expected changes in prices. Storage and other costs are assumed to be negligible for the sake of simplicity, we arrive at the following:

$$
\begin{aligned}
Q_{t} & =a p_{t}^{e}+\varepsilon x_{t}, \\
I_{t} & =b\left(p_{t+1}^{e}-p_{t}\right), \\
C_{t} & =-c p_{t},
\end{aligned}
$$

where $a, b, c$ and $\varepsilon$ are positive constants, $x_{t}$ represents the effect of exogenous factor (such as the weather) on supply and all the variables are measured as deviations from equilibrium.

Without loss of generality, we let $\varepsilon=0$. The model is completed with a market clearing condition: $C_{t+1}+I_{t+1}-\left(Q_{t+1}+I_{t}\right)=0$.

It is assumed that expectation is rational, which means perfect foresight in a deterministic context: $p_{t}^{e}=p_{t}$. Without loss of generality, let $\varepsilon=0$, the production and inventory determining processes are then given by:

$$
\begin{aligned}
Q_{t+1} & =f_{1}\left(Q_{t}, I_{t}\right)=Q_{t}+\alpha I_{t}, \\
I_{t+1} & =f_{2}\left(Q_{t}, I_{t}\right)=\beta Q_{t}+(1+\alpha \beta) I_{t},
\end{aligned}
$$

where $\alpha=(a / b)>0$ and $\beta=1+(c / a)>1$.

Now the Jacobian matrix is

$$
\mathcal{J}=\left(\begin{array}{cc}
1 & \alpha \\
\beta & 1+\alpha \beta
\end{array}\right)
$$

The eigenvalues will be a positive reciprocal pair due to the facts that

$$
\lambda_{1} \lambda_{2}=\mathcal{D}=1,
$$

and

$$
\lambda_{1}+\lambda_{2}=\mathcal{T}=2+\alpha \beta,
$$

which suggests that the fixed point $(0,0)$ is a typeIII fixed point.

If the stability is pursued with adaptive adjustment so that the production and inventory are adjusted with speed of $\gamma_{1}$ and $\gamma_{2}$, respectively,

$$
\begin{aligned}
Q_{t+1} & =\left(1-\gamma_{1}\right)\left(Q_{t}+\alpha I_{t}\right)+\gamma_{1} Q_{t}, \\
I_{t+1} & =\left(1-\gamma_{2}\right)\left(\beta Q_{t}+(1+\alpha \beta) I_{t}\right)+\gamma_{2} I_{t},
\end{aligned}
$$

The Jacobian matrix is adjusted to

$$
\tilde{\mathcal{J}}=\left(\begin{array}{cc}
1 & \left(1-\gamma_{1}\right) \alpha \\
\left(1-\gamma_{2}\right) \beta & 1+\left(1-\gamma_{2}\right) \alpha \beta
\end{array}\right) .
$$

Let $\delta=\alpha \beta$, we have $\tilde{D}=|\tilde{\mathcal{J}}|=1+\delta_{\gamma_{1}}\left(1-\gamma_{2}\right)$. Therefore, $\tilde{D}>1$ if $\gamma_{1} \gamma_{2}=0$, which implies the neither producer nor inventory keeper alone has 
enough power to force the process converge to the equilibrium. The stability can only be achieved when the producer is taking an adjustment speed in the conventional range, while the inventory keep takes it in the generalized range.

Stabilization regime is jointly given by flip bifurcation boundary $\left(\tilde{\lambda}_{2}=-1\right): \tilde{\mathcal{T}}+\tilde{\mathcal{D}}=-1$, and $\gamma_{2}>1$, where $\tilde{\mathcal{T}}=2+\left(1-\gamma_{2}\right) \delta$. A typical example is illustrated in Figure 7 for $\delta=2$.

This is an example of both economic agents are indispensable.

Finally, we provide an example in which both economic agents are dispensable, that is, either one is able to stabilize the market.

Example 6 None of adjustment parameters are indispensable (Puu (1997)): Consider a duopoly market where two firms produce identical goods, denoted by $x$ and $y$, with constant marginal cost $a$ and $b$, respectively. The market demand function is $1 /(x+y)$. It is assumed that both firms take Cournot strategy so that the each profit is maximized with the assumption that the output of its rival will not change, which gives rise to the so called Cournot reaction functions:

$$
x_{t+1}=\sqrt{\frac{y_{t}}{a}}-y_{t}, y_{t+1}=\sqrt{\frac{x_{t}}{b}}-x_{t} .
$$

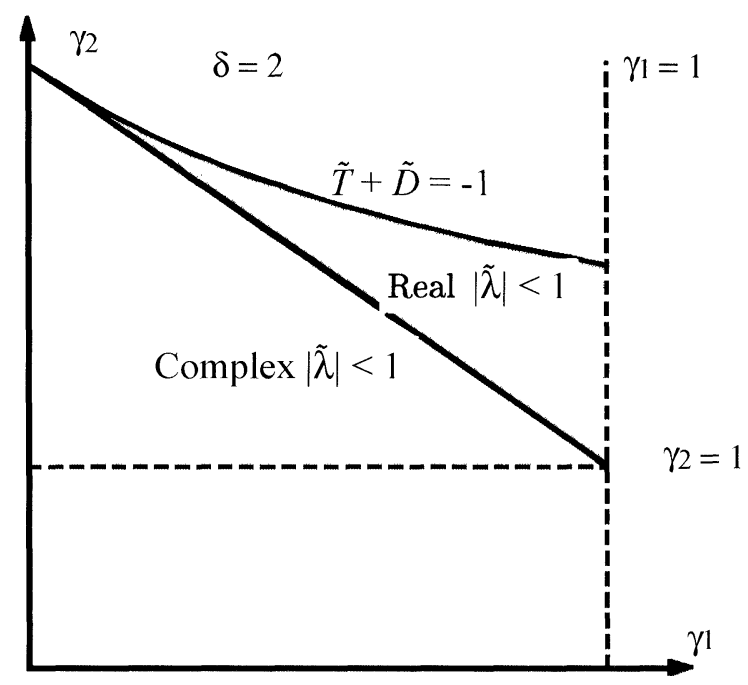

FIGURE 7 Asymmetric Stabilization.
At the equilibrium $\overline{\mathbf{X}}=(\bar{x}, \bar{y})=\left(\left(b /(a+b)^{2}\right)\right.$, $\left.\left(a /(a+b)^{2}\right)\right)$, the Jacobian matrix is

$$
\mathcal{J}(\overline{\mathbf{X}})=\left(\begin{array}{cc}
0 & \frac{1}{2(a+b)}-1 \\
\frac{1}{2(a+b)}-1 & 0
\end{array}\right)=\left(\begin{array}{ll}
0 & \lambda \\
\lambda & 0
\end{array}\right)
$$

with eigenvalues $\lambda_{1,2}= \pm \lambda= \pm((1 / 2(a+b))-1)$.

If $\lambda>1$, the fixed point $\overline{\mathbf{X}}$ is of type-III. If both firms decide to take adaptive adjustment with adjustment parameters $\gamma_{1}$ and $\gamma_{2}$ respectively, that is,

$$
\begin{aligned}
& x_{t+1}=\left(1-\gamma_{1}\right)\left(\sqrt{\frac{y_{t}}{a}}-y_{t}\right)+\gamma_{1} x_{t} \\
& y_{t+1}=\left(1-\gamma_{2}\right)\left(\sqrt{\frac{x_{t}}{b}}-x_{t}\right)+\gamma_{2} y_{t}
\end{aligned}
$$

then, at the same equilibrium $\overline{\mathbf{X}}$, the Jacobian becomes

$$
\tilde{\mathcal{J}}(\overline{\mathbf{X}})=\left(\begin{array}{cc}
\gamma_{1} & \left(1-\gamma_{1}\right) \lambda \\
\left(1-\gamma_{2}\right) \lambda & \gamma_{2}
\end{array}\right)
$$

which yields an eigenvalue pair:

$$
\begin{aligned}
\tilde{\lambda}_{1,2} & =\frac{1}{2}\left(\gamma_{1}+\gamma_{2}\right) \\
& \pm \frac{1}{2} \sqrt{\left(\gamma_{1}-\gamma_{2}\right)^{2}+4\left(1-\gamma_{1}\right)\left(1-\gamma_{2}\right) \lambda^{2}}
\end{aligned}
$$

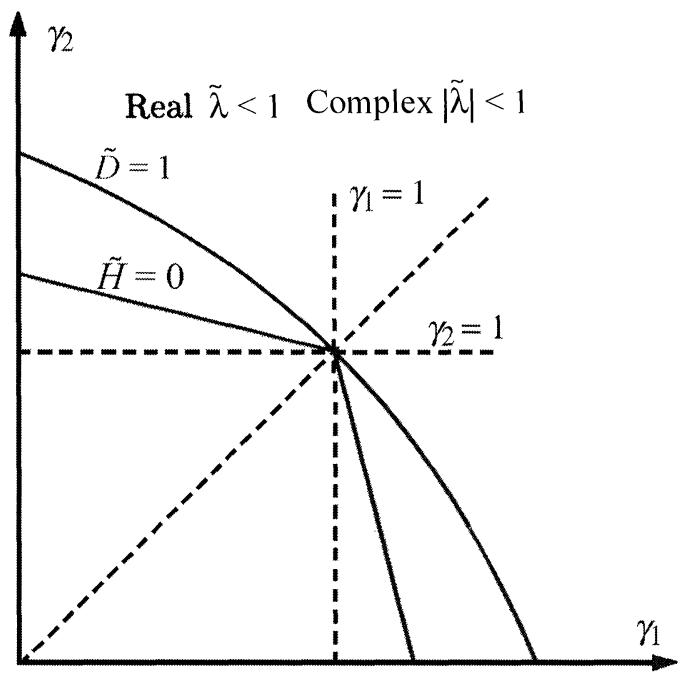

FIGURE 8 Symmetrical Stabilization. 
In this model, both firms have equal power in adjusting the market stability. So the stabilization regime in $\left(\gamma_{1}, \gamma_{2}\right)$ plane is symmetrical. Moreover, either firm alone can stabilize the market by taking an adaptive speed in the generalized range. A typical stabilization regime is illustrated in Figure 8.

\section{CONCLUDING REMARKS}

In this paper, Conventional adaptive expectations as a mechanism of stabilizing an unstable economic process is reexamined through a generalization to an adaptive adjustment framework. The generic structures of equilibria that can be stabilized through adaptive adjustment mechanisms are identified theoretical and numerically. The adaptive adjustment schemes so defined can be applied to a broader class of discrete economic processes where the variables interested can be adjusted or controlled directly by economic agents, such as in cobweb dynamics, Cournot games, Oligopoly markets, tatonnement price adjustment, tariff game, population control through immigration etc.

Comparing to other algorithms so far proposed in the natural sciences, the adaptive adjustment mechanism possesses some unique advantages. First, it requires neither a priori information about process nor any external generated control signal. Secondly, it is easy to implement in practice. Last but not least, it force the process to converge to its generic fixed points.

\section{References}

Chen, G. and Dong, X. (1998) From Chaos to Order, World Scientific, Singapore.

Chiarella, C. (1988) "The Cobweb Model, its Instability and the Onset of Chaos", Economic Modeling, 5, 377-384.

Day, R. H. (1994) Complex Economic Dynamics. Volume 1. MIT Press. Cambridge and London.
Fisher, F. M. (1961) "The Stability of the Cournot Oligopoly Solution: The Effects of Speeds of Adjustment and Increasing Marginal Costs", Review of Economic Studies, XXVIII(2), $125-135$.

Gandolfo, G. (1996) Economic Dynamics, (Springer-Verlag, Berlin).

Garratt, A. and Hall, S. G. (1997) "E-equilibria and adaptive expectations: Output and inflation in the LBS model", Journal. Economic Dynamics and Control, 21(7), 1149-1171.

Conlisk, J. (1993) "Adaptive Tactics in Games", Journal of Economic Behavior and Organization, 22(1), 51-68.

Heiner, R. A. (1989) "The Origin of Predictable Dynamic Behavior", Journal of Economic Behavior and Organization, 12, 233-257.

Heiner, R. A. (1992) "Adaptive Stability with Limited Structural Knowledge", Journal of Economic Behavior and Organization, 18, 123-126.

Hommes, C. H. (1991) "Adaptive Learning and Roads to Chaos, The Case of the Cobweb", Economic Letters, 36, $127-132$.

Huang, W. (1995) "Caution Implies Profit", Journal of Economic Behavior and Organization, 27, 257-277.

Marcet, A. and Sargent, T. J. (1988) "The Fate of Systems With Adaptive Expectations", The American Economic Review, 78-2, 168-172.

Mills, S. E. (1961) "The Use of Adaptive Expectations in Stability Analysis: A Comment", The Quarterly Journal of Economics, 75-2, 330-335.

Muth, J. F. (1961) "Rational Expectations and the Theory of Price Movements", Econometrica, 29, 315-335.

Naish, H. F. (1993) "The Near Optimality of Adaptive Expectations", Journal of Economic Behaviour and Organisation, 20, 3-22.

Nerlove, M. (1958) "Adaptive Expectations and Cobweb Phenomena", Quarterly Journal of Economics, 72, 227-240.

Okuguchi, K. (1970) "Adaptive Expectations in an Oligopoly Model", Review of Economic Studies, 36, 233-237.

Okuguchi, L. and Szidarovszky, F. (1990) The Theory of Oligopoly with Multi-Product Firms, (Springer-Verlag, Berlin).

Onozaki, T., Gernot, S. and Masanori, Y. (2000) "Complex Dynamics in a Cobweb Model with Adaptive Production Adjustment", Journal of Economic Behavior and Organization 41(2), $101-115$.

Puu, T. (1997) Nonlinear Economic Dynamics, (Springer-Verlag, Berlin).

Puu, T. (1998) "The Chaotic Duopolists Revisited", Journal of Economic Behavior and Organization, 33(3-4), 385-394.

Rassenti Stephen, Reynolds Stanley, S., Smith Vernon, L. and Szidarovszky Ferenc (2000) "Adaptation and Convergence of Behavior in Repeated Experimental Cournot Games", Journal of Economic Behavior and Organization, 41(2), $117-146$.

Szidarovszky, F. and Weiye, L. (2000) "A Note on the Stability of a Cournot-Nash Equilibrium: the Multiproduct Case with Adaptive Expectations", Journal of Mathematical Economics, 33(1), $101-107$.

Theocharis, R. D. (1960) "On the Stability of the Cournot Solution on the Oligopoly Problem", Review of Economic Studies, XXVII(2), No. 13, 133-4. 


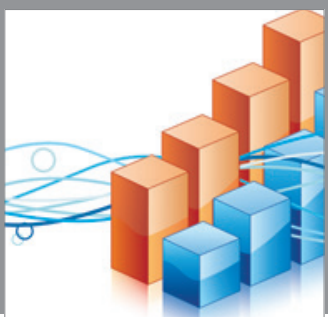

Advances in

Operations Research

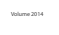

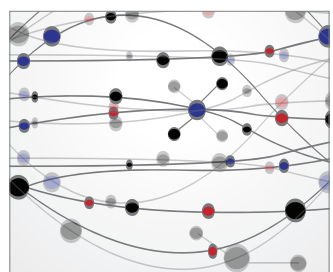

\section{The Scientific} World Journal
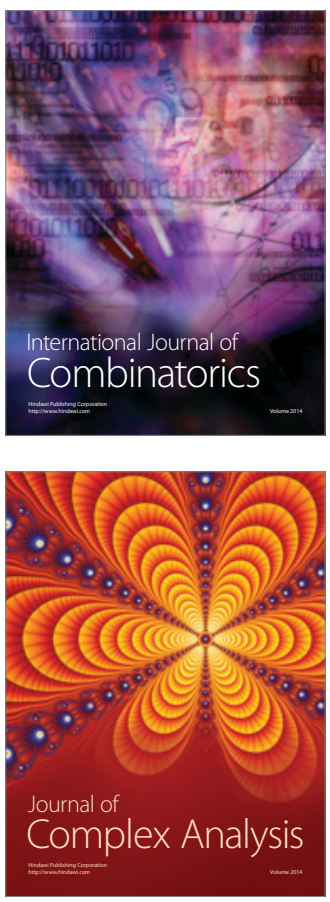

International Journal of

Mathematics and

Mathematical

Sciences
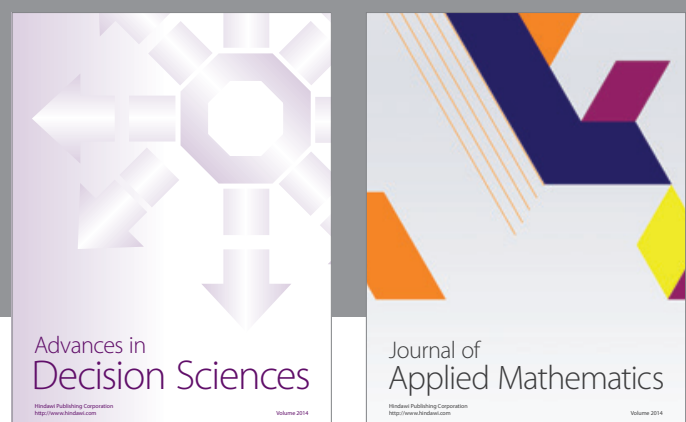

Journal of

Applied Mathematics
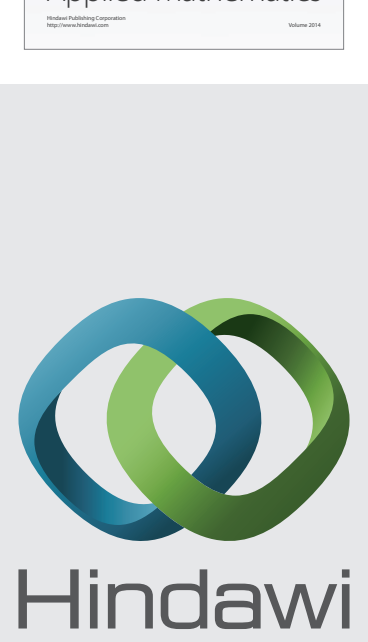

Submit your manuscripts at http://www.hindawi.com
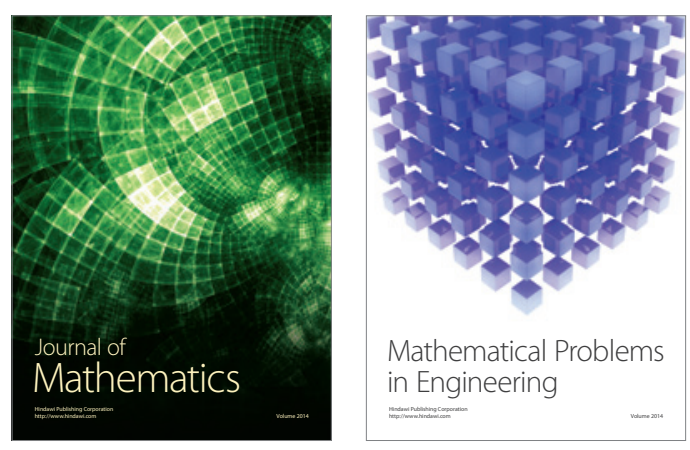

Mathematical Problems in Engineering
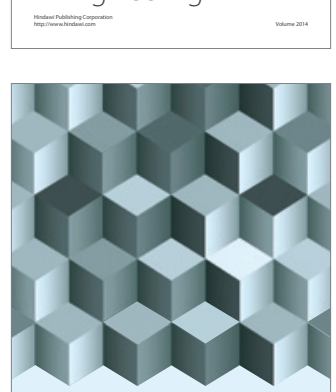

Journal of

Function Spaces
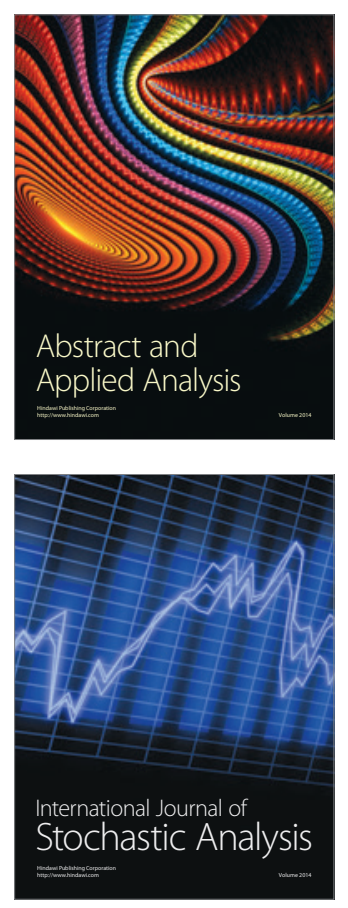

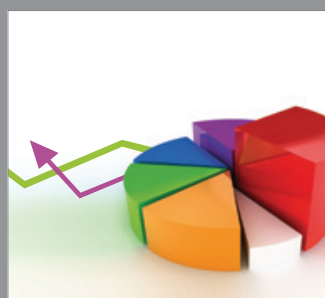

ournal of

Probability and Statistics

Promensencen
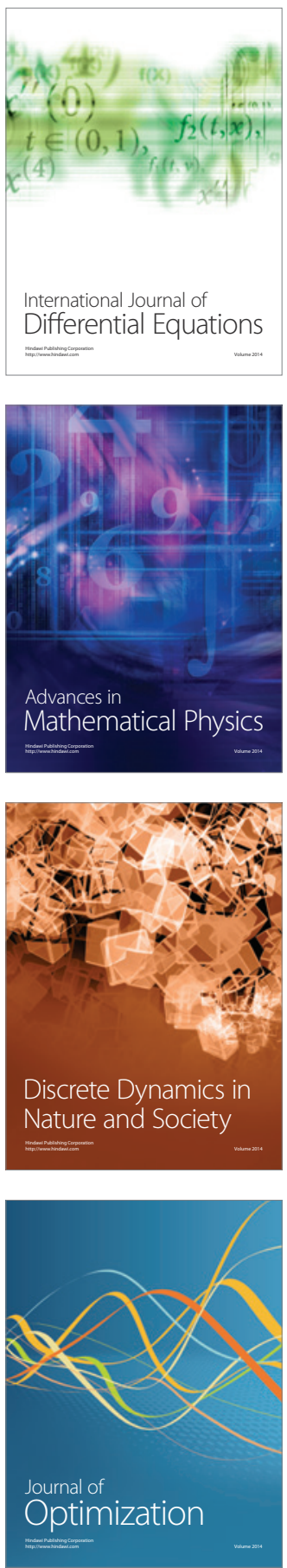